Arthroskopie $2019 \cdot 32: 307$

https://doi.org/10.1007/s00142-019-00303-y

(c) Springer Medizin Verlag GmbH, ein Teil von Springer Nature 2019

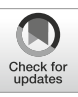

\author{
R. Seil ${ }^{1,2} \cdot$ M. T. Hirschmann ${ }^{3,4}$ \\ 'Centre Hospitalier de Luxembourg - Clinique d'Eich, Luxemburg, Luxemburg \\ ${ }^{2}$ Luxembourg Institute of Health, Luxemburg, Luxemburg \\ ${ }^{3}$ Department of Orthopaedic Surgery and Traumatology, Kantonsspital Baselland (Bruderholz, Liestal, \\ Laufen), Bruderholz, Schweiz \\ ${ }^{4}$ University of Basel, Basel, Schweiz
}

\title{
Bessere Ergebnisse durch offenen und ehrlichen Umgang mit Komplikationen
}

ist dies bereits der Fall, und sie werden mit einem solidaritätsbasierten Versicherungssystem auch finanziell abgefangen.

\section{) Wir werden mit unserer Erfahrung wachsen}

Der größte Lerneffekt kann erzielt werden, indem sich jeder Praktizierende intensiv mit seinen Problemen, Komplikationen und schlechten Ergebnissen auseinandersetzt. Wenn die Ursache der Probleme und Komplikationen besser verstanden und die notwendigen Schlüsse für die klinische Praxis wird, dann werden wir mit unserer Erfahrung wachsen - zum Wohl unserer Patienten. Nun muss nicht jeder von uns alle Erfahrungen selbst machen, es genügt auch, von geschätzten und erfahrenen Kollegen zu lernen.

Das hier vorliegende Themenheft beschäftigt sich daher ausschließlich mit der Frage, wie man mit Komplikationen und Problemen umgehen kann, zeigt Lösungen auf und diskutiert, wie diese vermieden werden können. In dieser Ausgabe der Arthroskopie werden die wichtigsten rekonstruktiven (z.B. Meniskus-, Kreuzbandoperationen, $\mathrm{Pa}$ tellastabilisierungen, Beinachsenkorrekturen) sowie teilprothetischen Lösungen von einer ausgewiesenen Autorenschaft mit großer Expertise in ihrem Fachgebiet behandelt. Lösungsvorschläge für die Behandlung von intra- und post- operativen Komplikationen werden im Detail aufgezeigt.

Wir hoffen, dass Ihnen das Themenheft zum Umgang mit Komplikationen gefällt und dass Sie aus den Beiträgen einige Tipps und Tricks für Ihre tägliche Arbeit entnehmen können.

Roman Seil

Michael T. Hirschmann

\section{Korrespondenzadresse}

Prof. Dr. med. M. T. Hirschmann

Department of Orthopaedic Surgery and Traumatology, Kantonsspital Baselland

(Bruderholz, Liestal, Laufen)

4101 Bruderholz, Schweiz

michael.hirschmann@ksbl.ch

michael.hirschmann@unibas.ch

Interessenkonflikt. R. Seil und M.T. Hirschmann geben an, dass kein Interessenkonflikt besteht. 